\title{
A triple-modality ultrasound computed tomography based on full-waveform data for industrial processes
}

This paper was downloaded from TechRxiv (https://www.techrxiv.org).

LICENSE

CC BY 4.0

SUBMISSION DATE / POSTED DATE

23-04-2021 / 25-06-2021

\section{CITATION}

Koulountzios, Panos; Rymarczyk, Tomasz; Soleimani, Manuchehr (2021): A triple-modality ultrasound computed tomography based on full-waveform data for industrial processes. TechRxiv. Preprint. https://doi.org/10.36227/techrxiv.14473341.v2

$\mathrm{DOI}$

10.36227/techrxiv.14473341.v2 


\title{
A triple-modality ultrasound computed tomography based on full-waveform data for industrial processes
}

\author{
Panagiotis Koulountzios ${ }^{1}$, Tomasz Rymarczyk ${ }^{2}$, and Manuchehr Soleimani ${ }^{1}$
}

\begin{abstract}
Ultrasound computed tomography (USCT) is gaining interests in many application areas in industrial processes. The recent scientific research focuses on the possible uses of USCT for varied fields of industry such as flow monitoring in pipes, non-destructive inspection, and monitoring of stirred tanks chemical processes. Until now most transmission tomography (UTT) and reflection tomography (URT) have been demonstrated individually for these applications. A full waveform USCT contain large amount of information on process under evaluation. The developed approach in this paper is focusing on demonstration of a triple modality USCT. First, an optimised transmission image is formed by fusion of images of from time-of-flight (TOF) transmission and acoustic attenuation (AA) images. Secondly, a reflection image is created informed by the combined transmission image. This triple modality approach enables integration of a shape-based approach obtained by URT mode with the quantitative image-based approach UTT mode. A delicate combination of the different information provided by various features of the full-wave signal offers optimal and increased spatial resolution and provides complementary information. Verification tests have been implemented using experimental phantoms of different combinations, sizes, and shapes, to investigate the qualitative imaging features. Moreover, experiments with different concentrations solutions further validate the quantitative traits to benefit from both reflection and transmission modes. This work displays the potential of the full-waveform USCT for industrial applications.
\end{abstract}

Index Terms - Ultrasound Computed Tomography (USCT); Ultrasound Process Tomography (UPT); Industrial processes; multi-modality ultrasound tomography; TOF imaging; AA imaging; Reflection imaging; full-waveform rich tomography

\section{Introduction}

$\mathrm{U}$ ltrasound computed tomography (USCT) has been studied lately on a broad spectrum of industrial applications with significant success [1-10]. Its usage has drawn a special attention notably relating to the imaging of biphasic medium and liquid mixtures in pipe flows and stirred reactors environments [11-15]. USCT works by analysing the acoustic wave propagation, via sound velocity or pulse amplitude decays of different materials. It aims to the mapping of medium's acoustical properties. It is non-invasive and non-destructive, compatible with high dynamical processes, like oil and gas flow. A better understanding of the measurement process and fast reconstructions algorithms are imperative for the use of

Paper submitted on 25th April 2021. This work was supported by the European Union's Horizon 2020 research and innovation programme under the Marie Skłodowska-Curie Grant 764902.

The authors would like to thank Dr. Dana Beiki (University of Bath) for proofreading the manuscript.

P. Koulountzios is with the Electrical and Electronic Engineering Department, University of Bath, UK. (email: P.koulountzios@bath.ac.uk)
USCT within the industry. Moreover, complex stirred tank processes, require sophisticated algorithms, which can provide accurate results. Due to the complex physical behaviour of acoustics propagation, there are multiple reconstruction modes, which use different waveform's properties. The transmission and reflection modes have been traditionally used in ultrasound tomography reconstructions, accounting for transmitted diffracted and reflected waves. Functional features of these reconstruction modes can be complementary. For instance, reflection tomography offers good resolution at the boundary of different media, while the transmission method has better resolution in distinguishing discontinuities along the signals' propagation path. Transmission mode properties, such as

Tomasz Rymarczyk is with Research \& Development Centre Netrix S.A., Wojciechowska 31, 20-704 Lublin, Poland. (Email: tomasz@rymarczyk.com)

M. Soleimani is with the Electrical and Electronic Engineering Department, University of Bath, UK. (email: M.Soleimani@bath.ac.uk) 
acoustic attenuation (AA) or the time-of-flight (TOF), can be used to determine the amplitude and sound-speed profiles of the region of interest (ROI), offering quantitative information. All the AA, TOF, and reflection modes have drawbacks with artefacts under certain biphasic medium distributions. Thus, the performance of single-modality Ultrasonic Process Tomography (UPT) is limited. However, these shortcomings may be compensated with a multi-modality reconstruction. For instance, in a liquid-liquid mixture, the reflected signals will be significantly low, leading to a possible reflection reconstruction failure. However, transmission image should be more meaningful in such scenarios. On the other hand, in a liquidsolid or liquid-gas medium, reflected waves may be exploited. For instance, in many stirred tanks chemical processes like fermentation and crystallization, localised super-saturated suspensions may be formed due to process malfunction e.g., stirrer malfunction. In this case, a drastic structural phase difference may occur. Thereafter, reflection mode might be used as a malfunction detection by detecting the localised high concentrated suspensions. It would be also possible to detect complex dynamic phenomena such as a gas-flow or vortexes coming from a high stirring effect. Although, the use of reflection mode in that direction needs to be further investigated.

The multiple mechanisms (transmission, diffraction, reflection) during ultrasound propagation and the rich information (attenuation, time-delay, distortion) contained in an ultrasonic full signal, establishes the need for a multi-modality method. Therefore, a multi-modality approach, that can facilitate multiple reconstruction methods, is expected to result in more accurate imaging, as it can process measurements coming from different signal's features. Several studies show that dual-modality UPT (transmission/reflection) is superior to single-modality, confirming that multi-modality offers tremendous benefits [16], [17]. As a result, a novel triplemodality image reconstruction method combining AA and TOF transmission as well as TOF reflection is proposed.

In a small circular setup filled with a non-homogeneous medium, significant back-scattering and reflections are expected to happen. Thus, the "noise" levels are higher. Most common issues in ultrasound tomography revolve around the estimation of TOF and AA from the full-waveforms, especially in instruments that are not calibrated or that are characterised by high "noise" levels [18], [19], [20], [21]. A novel reflection TOF picking method was built to tackle this problem. It exploits a forward reflection solver based on ray acoustics, to optimise the recorded reflected TOF values and subsequently the reflected image. The proposed method fits well in the robust triple-modality proposed approach. The amplitude of the transmitted pulses and time-of-flight of both the transmitted and reflected pulses have been used to produce three different reconstructions (TOF, AA, reflection). Finally, a method of image fusion, using the results of TOF-UTT, AA-UTT and URT methods, was developed and used to generate the final image.

The paper is organised as follows. Section II presents the main functionality of the tomographic system and details its specifications. Moreover, the methods undertaken for transmitted and reflected TOF and AA picking are further characterised. Section III describes the reconstruction formulas for transmission and reflection tomography, while section IV presents the proposed algorithm for reflected TOF picking, which helps optimising the recordings. Section V presents the developed fusion method. Finally, in section VI, the experimental results are presented and evaluated, and in section VII, the conclusions and discussion occur.

\section{MEASURING SYSTEM}

Figure 1 depicts the design of such a triple-modality ultrasound tomographic concept. For a 16-channel transducer system each sensor acts as both transmitter and receiver and complete tomographic data is collected by exciting each sensor in turn. When $\mathrm{Tx} 1$ is an excitation transducer $\mathrm{Rx} 1$, $\mathrm{Rx} 2, \ldots, \mathrm{Rx} 15$ represent the receivers. The transmission mode uses AA and TOF data for Rx3, Rx4, Rx5, ..., Rx13 and for reflection mode $\mathrm{Rx} 1, \mathrm{Rx} 2, \mathrm{Rx} 14, \mathrm{Rx} 15$ data are used. The actuated receivers in transmission mode are those that are included in the fan beam of 120-degree angle where the best quality of transmission data is possible. Those, located in the neighbourhood of the transmitter are excluded from transmission mode data but used in reflection mode.

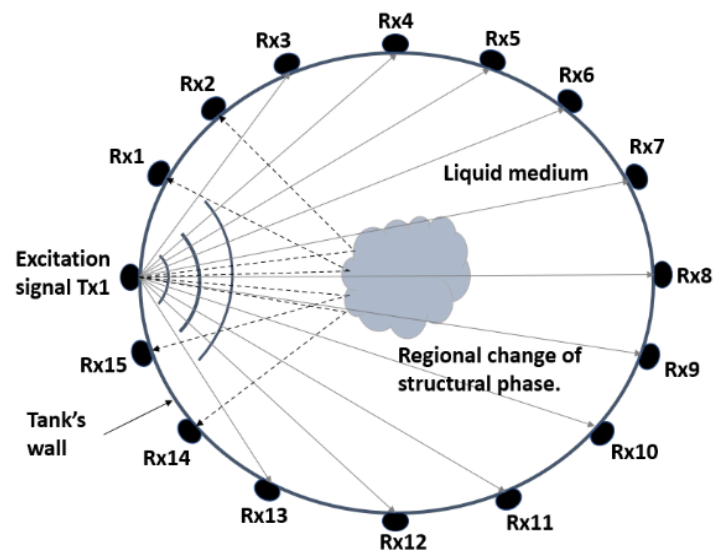

Figure 1. Triple-modality ultrasonic tomography (transmission/ reflection). Design of transmitted and reflected signals' paths.

The multi-modality USCT approach utilizes TOF and amplitude information from transmission and reflection waves. These waves come from the interaction of different phase structure within the medium. Acoustical properties are dependent on changes in the structural phases (i.e., acoustic impedance, velocity). Sound transmission and reflection would, therefore, change within these phases. Acoustic impedance $\mathrm{Z}$ affects the propagation and nature of pulse excitation and is dependent on the material's structural phase. It indicates the intensity of a medium's regions to block the vibrations of the particles in the acoustic field [22]. The ratio of the reflected pulse's amplitude, $P_{r}$, to the incident wave's amplitude, $P_{o}$, is called the acoustic pressure reflection coefficient $\mathrm{R}$ [23], and it is defined as:

$$
R=\frac{P_{r}}{P_{o}}=\frac{Z_{1} \cos \theta_{0}-Z_{2} \cos \theta_{t}}{Z_{1} \cos \theta_{0}+Z_{2} \cos \theta_{t}}
$$


By the same way, the acoustic pressure transmission coefficient, $\mathrm{T}$, is defined as:

$$
T=\frac{P_{r}}{P_{0}}=\frac{2 Z_{1} \cos \theta_{0}}{Z_{1} \cos \theta_{0}+Z_{2} \cos \theta_{t}}
$$

Where $P_{r}, P_{t}$ define the reflection and transmitted wave's acoustic pressure; $\theta_{0}, \theta_{t}$ represent the wave's angle of incidence and angle of transmission, respectively; $P_{0}$ is the acoustic pressure of the incident wave. $Z_{1}$ and $Z_{2}$ are the acoustic impedances of medium 1 and medium 2 .

When a sound wave propagates through a medium, its intensity decreases with the distance travelled, as expressed in eq. (3).

$$
A m=A m_{0} e^{-\mu h}
$$

Where $\mathrm{Am}_{0}$ is the amplitude of the propagating wave at a given location. Am is the reduced amplitude at another location. In this case, $h$ is the distance travelled between the two locations, and $\mu$ is the attenuation coefficient in Neper $(\mathrm{Np}) /$ length. The two primary mechanisms that cause the attenuation of sound energy are absorption and scattering. Industrial processes usually consist of multiple phase media with a drastic difference in structural phase. Such conditions are favourable for a multimodality approach in ultrasonic reconstructions, exploiting attenuation, sound-speed and acoustic impedance change within the medium.

\section{A. Tomographic device}

The ultrasonic tomograph has 32 independently working channels that can perform measurements in transmission and reflection modes. In this paper, we use 16 channel sensors in a single plan 2D USCT mode. The bottom layer was used for the data collection. At the same time, a sensor sends an ultrasonic signal of 5 cycles (tone burst), while remaining sensors record. Respectively, receivers measure the full-waveform signal. The sequence repeats until every sensor produces an excitation signal. The system inside the reinforced suitcase consists of eight four-channel measurement cards connected via a FD CAN bus to the measurement module. The measuring module is a bridge between a microprocessor measuring system with a touch panel or external control application (Figure 2). Each device channel has its own analogue signal processing module and its own 12-bit ADC 4MSPS converter. In TOF and amplitude measurement mode, the signal is normalised to voltages from $0-3.3 \mathrm{~V}$, because each transducer has a reference voltage of $3.3 \mathrm{~V}$. The sampling frequency is 0.25 samples per micro-second, which results directly from the ADC converter speed. A Built-in envelope converter was used for converting an analogue acoustic signal to the envelope with the possibility of switching its configuration to a frequency of $400 \mathrm{kHz}$. In addition, the measurement module can monitor the measuring sequence, store user-specific parameters, control the high voltage inverter, and switch the USB HS bus between the socket in the front panel and a touch panel. The touch panel was made using a RaspberryPi 4B 2GB RAM board and a 7-inch capacitive touch screen. The most crucial data buses have been led to the front panel of the device.

\section{B. TOF/ AA picking method for transmission tomography}

Transmission signals directly travel from the transmitter to the receivers without any reflection. These signals undergo either diffraction or direct transmission, without significant change of direction. A transmission pulse usually travels faster and transmits at a larger amplitude. Figure 3(a) shows a fullwaveform signal and its envelope, recorded by Rx6 upon Tx1 excitation.
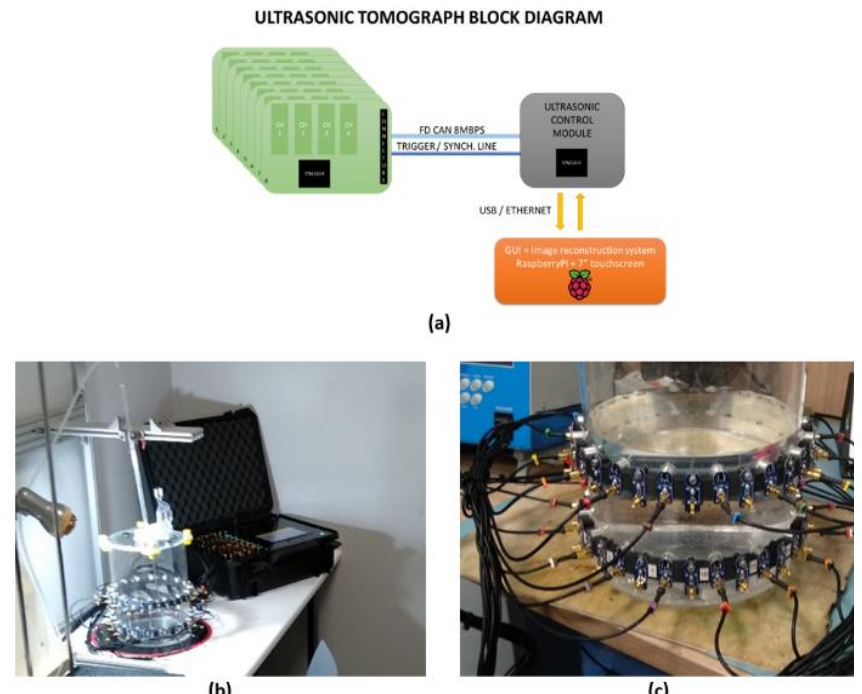

Figure 2. (a) Measurement system: Ultrasonic tomograph block diagram. (b) ultrasound tomographic system. (c) tank with sensors.

Moreover, it illustrates signal specific TOF and AA picked points, calculated by the applied method. Figure 3(b), (c) present background and full calculated TOF and amplitude data. The picking method of transmitted TOF values is described below.

First, the analytic envelope of the signal processed using Hilbert transform. Using the envelope, signal oscillation can be removed, facilitating more accurate peak detection. The advantage of using the envelope is to migrate the effect of arbitrariness and to eliminate the effect of phase changes. Then, the enveloped is processed to detect the transmitted pulse and record its $\mathrm{x}$-value, defining the pulse's travel-time, and y-value, determining the recorded pressure. The method is described in eq. (4-8), where $v(i)$ represents an enveloped signal, in our experiments the receiving signal contains 1665 samples or time steps. A minimum threshold of $10 \%$, th, is used to cut down the minor pulses caused by back-scattering or equipment-related noise, eq. (4). TOF value is determined by the projection of the first signal's point after threshold, th, to the x-axis. According to the sampling frequency, the TOF must be multiplied by 0.25 , to be converted in $\mu$ sec. Eq. (5-7) describe this in a linearised fashion. Comparatively, the biggest y-value within a $20 \%$ signal's window in the transmitted pulse "region" indicates the recorded pulse's amplitude, eq. (8).

$$
\text { th }=0.1 \max (v(i)) \text {, where } i=\{1,2, \ldots, n\}, q=1665
$$




$$
\begin{gathered}
v^{\prime}(i)=\left\{\begin{array}{c}
v(i) \text { for } v(i)>\text { th } \\
0 \quad \text { for } v(i)<\text { th }
\end{array}\right. \\
k_{j}=i \mid v^{\prime}(i) \neq 0, j=\{1,2, \ldots, m\} \text { where } m \leq n \\
\text { TOF }=0.25 k_{1} \\
A A=\max \left(v^{\prime}(l)\right), \\
\text { where }\{l=k \mid l=\{i, i+1, \ldots, i+0.2 q\}
\end{gathered}
$$

All reconstructions are generated by using difference imaging, collecting background data (reference data), $\mathrm{TOF}_{\mathrm{back}}$, and full data (data collected by scanning a non-uniform medium), $\mathrm{TOF}_{\text {full }}$. TOF measurement data, $\mathrm{TOF}_{\mathrm{tr}}$, originates from the subtraction of full data from the background data and define the travel-time delays ( $\mu \mathrm{s})$, eq. (9).

$$
\begin{gathered}
\mathrm{TOF}_{\mathrm{tr}}=\mathrm{TOF}_{\text {back }-\mathrm{TOF}_{\text {full }} \text { where }} \\
\mathrm{TOF}_{\mathrm{tr}}\left\{\begin{array}{l}
0 \quad \text { for } \mathrm{TOF}_{\text {tr }}<0 \\
\mathrm{TOF}_{\text {tr }} \text { for } \mathrm{TOF}_{\mathrm{tr}}>0
\end{array}\right.
\end{gathered}
$$

AA measurement data are computed by eq. (10) [24].

$$
A A_{\text {tr }}=\frac{1}{f_{c}} \ln \left(\frac{A A_{\text {back }}}{A A_{\text {full }}}\right)
$$

Where $A A_{\text {back }}$ is the signal's amplitude at each receiver when there is only water (reference data) in the field of view (FOV) and $A A_{\text {full }}$ is the amplitude of the full data. $f_{c}$ is the centre frequency of the excitation pulse.

In both TOF and amplitude data, the "Deleting Outliers" statistical filtering method was used to handle this noise for all the datasets [25]. Specifically, "outlier" TOF values usually are generated from back-scattering or reflected signals. Iterative implementation of the Grubbs Test was used to identify the outlier signals. In any given iteration, the tested value is either the highest or lowest value, represented by the furthest value from the sample mean.

\section{TOF picking method for reflection tomography}

The "traditional" picking method of reflected pulses is described in this section. This method makes full use of all the four transducers poisoned in pairs, on each side of the emitter. Contrasting full and background measurements, cancels tankrelated back-scattering and reflection, as expressed in eq. (11). As both background and full data are assumed to present similar tank specific back-scattering and reflection. The recorded maximum pulse peak is assumed as the observed reflected TOF, $T O F_{r f l}$, as shown in eq. (12).

$$
\begin{gathered}
p_{i}=\left|\left(v(i)_{\text {back }}-v(i)_{\text {full }}\right)\right|, \\
\text { where } i=\{1,2, \ldots, 1665\} \\
\text { TOF }_{r f}^{\text {obs }}=\max \left(p_{i}\right), \text { where } i=\{1,2, \ldots, 900\}
\end{gathered}
$$

Figure 4(a) illustrates the experimental setting. Figure 4(b) represents the plots of full (background and inclusions) and background waveforms and their respective envelopes. The first peak comes from the transmission pulse. The second peak comes from the reflected pulse within the inclusion surface Lastly, the third peak represents tank-related back-scattered signal. Absolute subtraction optimises reflected pulses measurements, reducing overall noise and back-scattered effects. As the reflected pulse remain unchanged, it is easily trackable. Figure 4(c) depicts the absolute subtracted signal.

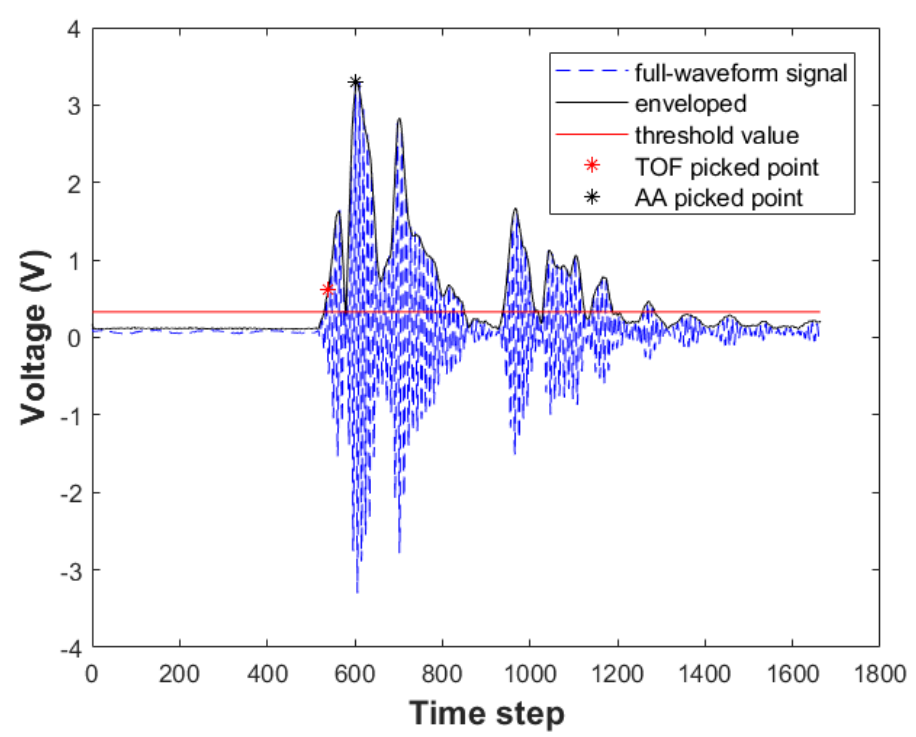

(a)

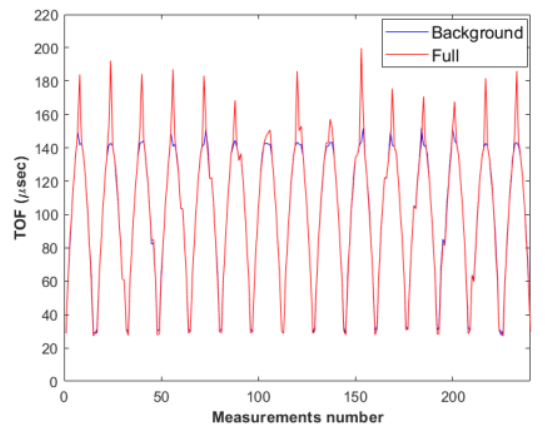

(b)

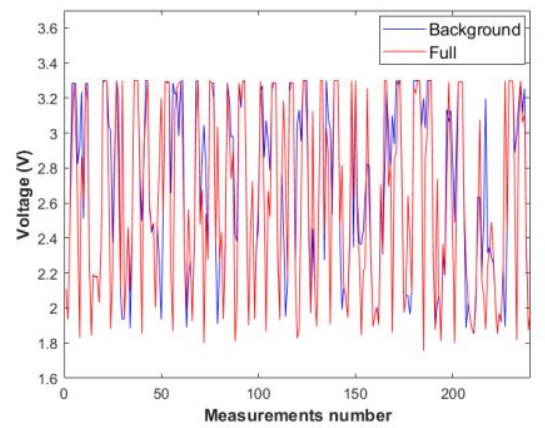

(c)

Figure 3. (a) Recorded full-waveform signals from Tx1-Rx6 pair, with its envelope. Each timestep is $1 / 4 \mu$ sec. (b) TOF data computed from the enveloped signals. (c) AA data calculated from the enveloped signals. 


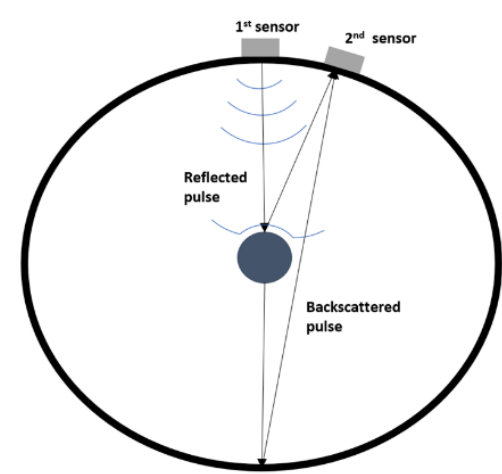

(a)

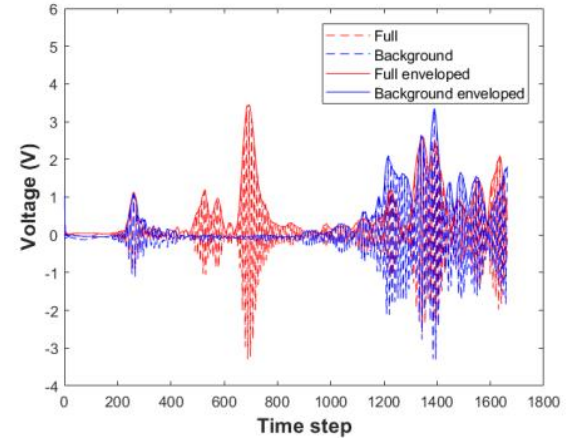

(b)

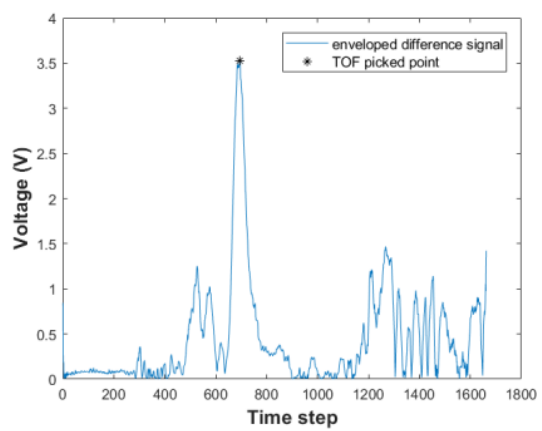

(c)

Figure 4. (a) Schematic of the setup. (b) Background and Full measurements in full waveforms. (c) Difference data in full waveforms. Each timestep is $1 / 4 \mu$ sec.

\section{MEthODS}

\section{A. Transmission mode reconstruction}

Transmission can be measured either via a travel-time or an acoustic attenuation technique. Transmission reconstruction would take place via either travel time or the amplitude decay of the first-arrival pulse [26]. The most used approximation for transmission USCT is the ray-based method. It is fundamental in most tomographic schemes, as the line integral defines the path of a high frequency propagating pulse between an emitter and a receiver. It is a simplified approach, which does not account for the diffraction effect caused by non-homogeneous medium. Therefore, a computational model based on diffraction on the 1st Fresnel zone [27]. Fresnel volume or 'fat ray' tomography is an appealing compromise between the efficient ray theory tomography and the computationally intensive full waveform tomography [28]. Using a finite frequency approximation to the wave equation leads to a sensitivity kernel where the sensitivity of the travel time delay also appears in a zone around the fastest ray path. The delay time is given as:

$$
\Delta t(x)=t(s, x)+t(x, r)-t_{0}(s, r)
$$

Here $\mathrm{t}(\mathrm{s}, \mathrm{x})$ and $\mathrm{t}(\mathrm{x}, \mathrm{r})$ are the travel time from the source (s) to $\mathrm{x}$ and from $\mathrm{x}$ to the receiver ( $\mathrm{r})$ and $t_{0}(s, r)$ is the travel time along the ray path from the source to receiver. The times of travelling can be evaluated using the ray-tracing method. A point $\mathrm{x}$ is always within the first Fresnel zone if the corresponding travel-time satisfies the eq. (14), in which T defines the emitted wave's period:

$$
|\Delta t(x)|<\frac{T}{4}
$$

The following function defines the sensitivity of a Frechet kernel based on the first Fresnel zone:

$$
\begin{aligned}
& S(x) \\
& =K \mathrm{~V}(s, x) \mathrm{V}(x, r) \cos \left(2 \pi \frac{\Delta t(x)}{T}\right) \exp \left(-\left(\frac{a \Delta t(x)}{\frac{T}{4}}\right)^{2}\right)
\end{aligned}
$$

Where $S(x)$ is the sensitivity at $x, \mathrm{~V}(s, x)$ and $\mathrm{V}(x, r)$ are the amplitude values of the wavefield at $x$ propagating from $s$ to $x$ and from $x$ to $r$, respectively, and $\mathrm{k}$ is a constant value for normalisation purpose. The cosine factor models the alternating sensitivity being positive in the odd Fresnel zones and negative in the even Fresnel zones. The $a$, in the Gaussian factor controls the degree of cancellation in Fresnel zones. The geometrical spreading approximates the amplitude factors in a homogeneous medium. The normalisation of the kernels is achieved by ensuring that the integrated sensitivity over the whole medium is equal to the length of the reference ray path [29]. SIPPI MATLAB software has been used to generate these sensitivity kernels [30]. These kernels represent the acoustic distribution of the medium of each sensor's excitation, forming the sensitivity matrix. A Normalisation method based on the geometric wave path was applied to the generated kernels to ensure an accurate time-of-flight (TOF) and acousticattenuation (AA) mapping, as described in eq. (16).

$$
A_{i, j}=\frac{H_{i, j}}{\sum_{i_{1}=1}^{m} \sum_{j_{1}=j} H_{i_{1}, j_{1}}}
$$

where $H_{i, j}$ is the sensitivity matrix based on the Frechet method and $A_{i, j}$ is the normalized matrix, used for reconstructions, and for $\mathrm{m}$ measured data and $\mathrm{n}$ number pixels, $\mathrm{i}=[1, \ldots \mathrm{m}]$ and $j=[1, \ldots, n]$.

A tomographic approach needs the transmission sensitivity matrix to simulate the propagation of the measured energy from sensors. The measurement data for transmission tomography includes TOF and AA data. The so-called forward problem is formed by the multiplication of the sensitivity matrix with the measurement data. Below the notation defines $\Delta M$ stands for both TOF and AA data for TOF and AA reconstructions, respectively. A generalised tomographic forward problem can be expressed as:

$$
\Delta M=\mathrm{A} \Delta S+e
$$

Where $\Delta S$ is the reconstructed distribution based on acoustic features, A is the modelling operator which expresses the sensitivity distribution in the FOV, $\Delta M$ is the sensor's recorded 
data, and $e$ is the noise in the measurements. A simplified inversion can be done using back projection.

$$
\Delta S \approx A^{T} \Delta M
$$

Total Variation regularisation (TV) [31], [32] was used, which has more significant potential in solving the regularised inverse problem in a stabilised fashion. The TV problem is defined as an optimisation problem, minimizing

$$
\left.\|A \Delta S-\Delta M\|\right|^{2}+\left.a|| \nabla \Delta S\right|_{1}
$$

Where $a$, the regularisation parameter, $\nabla$ is the gradient and $\|.\|_{1}$ is the $l_{1}$-norm. Then the problem to be solved is the constrained optimisation problem, as shown in eq. (20).

$$
\begin{array}{r}
x_{a}=\operatorname{argmin}_{\Delta S}\left(\alpha|| \nabla \Delta S||_{1}\right) \text { such that } \\
\|\mathrm{A} \Delta S-\Delta M\|^{2}<p \text {, where } \mathrm{p} \text { is determines }
\end{array}
$$

based on our knowledge of measurement noise

It is solved by the Split Bregman based TV algorithm [33], [34]. Then, carefully choosing the regularisation parameter, we optimise the image by deleting undesired artefacts.

\section{B. Reflection Reconstruction}

A time-of-flight reflection method applied to reconstruct the captured reflected pulse travel times. The objective of this method is to locate the reflection points, which lie between the interaction of the object's boundaries with the medium. Figure 5 (a) presents a geometric representation of the sensors and directly transmitted and reflected waves. Tx1 emits a tone burst pulse while $\mathrm{Rx} 1, \mathrm{Rx} 2, \mathrm{Rx} 14$, and $\mathrm{Rx} 15$ record the reflection signals. In this case, a relevant algorithm is developed to connect every Tx with its four Rx points. For instance, in Tx1$\mathrm{Rx} 1$, the algorithm connects the two points, finds the mid-point $\mathrm{P}$ of the line, then connects $\mathrm{P}$ to the centre of the circle (centre of the circular object), the intersection point $\mathrm{C}$ is the estimated reflection point. Using the coordinates of $\mathrm{C}$, one can compute the travelling distance of the pulse and subsequently the reflected TOF data. This method comprises the reflection forward problem and can be used to calculate simulated reflection TOF data.

To reconstruct the acoustic profile of the medium using captured reflected TOF data, a reflection reconstruction model based on an ellipse intersecting algorithm was used[35], [36], [37], [38]. If transmitter and receiver are different, the backprojection is an ellipsoidal locus with the ellipse's foci at transducer positions. The image is reconstructed by drawing arcs of an ellipse along the reflection path. Input TOF values are translated to the pulse's travelled distance by using the prior information of the sensors' coordinates, as shown in eq. (21).

$$
\begin{gathered}
d=s_{0} \text { TOF } F_{\text {refl }} \\
\text { where } d=d_{\mathrm{Tx} 1-\mathrm{C}}+d_{\mathrm{Rx} 1-\mathrm{C}}
\end{gathered}
$$
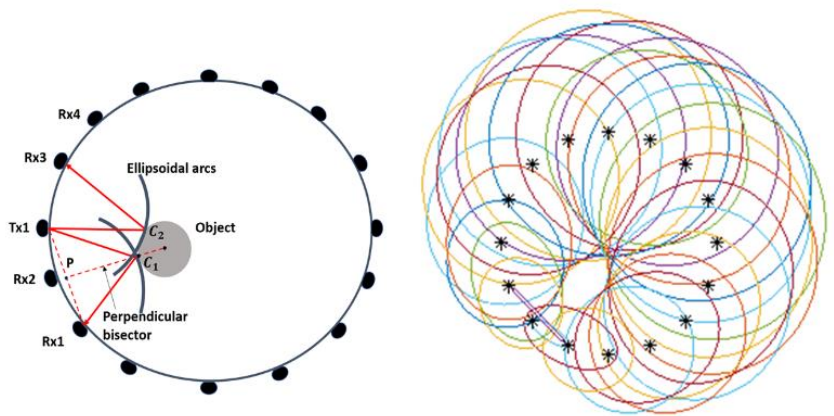

Figure 5. (a) Functionality of the reflection tomography and geometrical computation of reflection points $C_{1}$ and $C_{2}$. (b) Reflection image by superimposing all the ellipses.

Where $d_{\mathrm{Rx} 1-\mathrm{C}}$ and $d_{\mathrm{Tx} 1-\mathrm{C}}$ denote the axial distance between the reflection point and the receiver and between the reflection point and the transmitter, respectively. $T O F_{\text {refl }}$ represents the time of flight and $s_{0}$ is the sound speed in the water. Superimposing the arcs of ellipses generate an image where the intersection of these ellipses highlights the boundary of the circular object. The eq. (16) is used to produce all these ellipses that can define the edges of the medium that allow reflection:

$$
A C+C B=2 a=d
$$

$\mathrm{A}$ and $\mathrm{B}$ are two foci of the ellipse, and $\mathrm{C}$ is a point located in the ellipse curve, $a$ stands for the long axis length of the ellipse. $\mathrm{A}$ and $\mathrm{B}$ represent the transmitter and receiver; respectively, $\mathrm{C}$ stands for a particular point of the target surface, reflecting the ultrasound wave. The value of $a$ can be easily calculated, using also the ellipse equation of eq. (23).

$$
a^{2}=b^{2}+c^{2}
$$

The value of $b$ and $c$ can also be easily obtained where $b$ is the short axis length of the ellipse and $\mathrm{c}$ is the distance between focus and the ellipse centre. The distance can be calculated by the equation:

$$
c=\frac{1}{2} \sqrt{\left(x_{r}-x_{R}\right)^{2}+\left(y_{r}-y_{R}\right)^{2}}
$$

where $x_{r}, x_{R}, y_{r}, y_{R}$ are the transducer coordinates, their subscripts indicate the transducer mode. When all ellipse parameters are obtained, a particular ellipse can be drawn in a determined position and dimension. At last, the target image can be found by many ellipses that are mutually intersected. Figure 5 (b) presents ellipses generated by the developed reflection reconstruction program.

\section{NOVEL REFLECTION PULSE PICKING APPROACH AIDED BY TRANSMISSION RECONSTRUCTION}

Dual modality ultrasound imaging, fusing transmission and reflection reconstructions, have been recently researched as an optimised ultrasound tomography method [9], [16], [39]. 


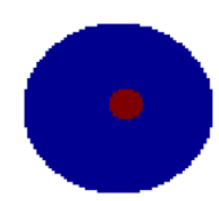

(a)

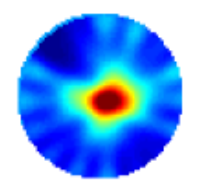

(b)

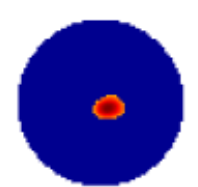

(c)

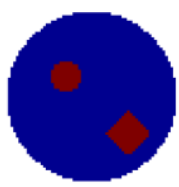

(d)

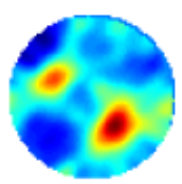

(e)

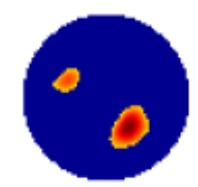

(f)

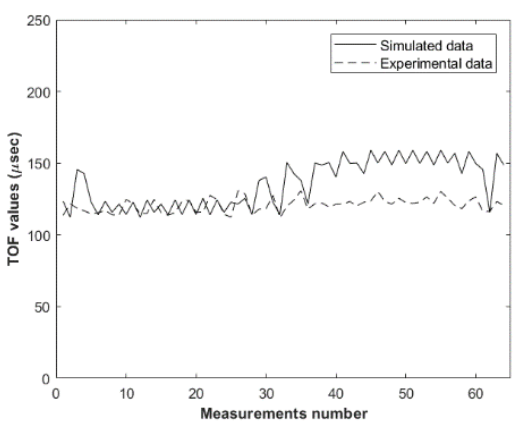

(g)

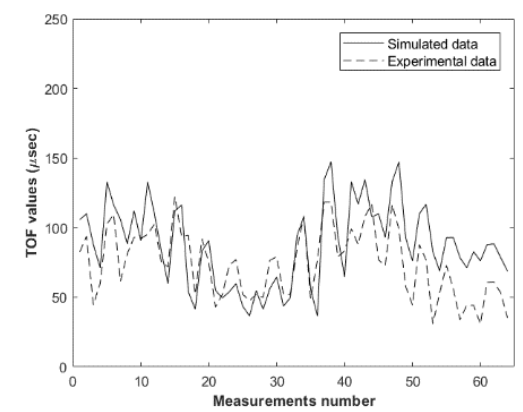

(h)

Figure 6. (a), (d) True images. (b), (e) Fused transmission images. (c), (f) Segmented Images used as domain in forward reflection solver. (g), (h) Reflection simulated data.

Indeed, combining the two different modalities, which use different full-waveform's features, can aid reconstructions by combining complementary information. Despite the good performance of transmission imaging, reflection imaging can aid more towards the improvement of outcomes, especially in well-characterizing the domain boundaries. Therefore, a robust algorithm for reflection reconstruction needs to exploit the medium's boundaries. In practice however, picking algorithms, few times, struggle to locate the correct reflection pulse, and noise is added to the measurements. This is a common issue of all ultrasonic tomographic instruments, caused by the backscattering effect [40]. Therefore, a reflection TOF picking method guided by transmission image was developed. The developed method is based on the reflection forward solver to produce better-reflected TOF values than those coming from picking the reflected pulses, described in section IV.

\section{A. Image segmentation \& Reflection forward solver}

An acoustic profile domain of the ROI is created by fused transmission image and used from the reflection forward solver to produce the simulated reflected data, $T O F_{r f}^{s i m}$. In that case, the fused transmission image is used as described below in Section V. A segmentation approach has been developed to define the acoustic distribution on the fused transmission image. First, the global Otsu's thresholding method was used [41]. Then, a labelling method calculates the number of noncontinuous detected regions [43]. For every region, the centre of mass and its boundaries' shape are calculated. The solver uses the regions' information to find the closest region to the corresponding pair of transmitter-receiver, to avoid multiple reflection signals. This aims to improve accuracy, notably considering the impact of more complex distributions on the reflective forward solver. Then, as shown in Figure 5(a), a line is computed from the middle point of sensors to the region's centre of mass.

The intersection of this line with the region's boundaries forms the intersection point $C$. Using eq. (22), given that $\mathrm{A}$ and $\mathrm{B}$ represent the two sensors, calculates the wave's travelled distance $d$. Finally, the simulated reflected data, $T O F_{r f}^{\text {sim }}$, are generated by eq. (25).

$$
T O F_{r f}^{\operatorname{sim}}=\frac{s_{0}}{d}
$$

Figure 6(a-f) present the true, transmission reconstructed and segmentation images of two single and double inclusions cases. Figure $6(\mathrm{~g}-\mathrm{h})$ present the recorded, $T O F_{r f}^{o b s}$, and the simulated, $T O F_{r f}^{s i m}$, reflection data, using the reflection forward solver. In the reflected data, there are lower values region for every positioned object, coming from the time-delays that they introduce. In Figure 6(g), a single low-values region can be noticed, while Figure 6(h) two of them. Furthermore, a clear resemblance between simulated and observed data can be noticed, showing the good performance of the reflection forward solver. The number of reflection points reduces significantly in multiple inclusions cases, which is clearly a disadvantage of the method. However, a ring setup with more sensors would increase the spatial resolution and the accuracy of the method.

\section{B. Minimum distances method}

This method incorporates an optimised travel-time picking method. It picks a signal's value, using as an a priori information the simulated reflection data, $T O F_{r f}^{\text {sim }}$, to optimise the observed data, $T O F_{r f}^{o b s}$. At first, an appropriate threshold was set, and all the potential reflected peaks above that threshold were stored, $\left(P_{m, n}\right)$. In this way, no peak is being excluded. Then, the algorithm calculates the "closest" peak to the corresponding simulated TOF value, $T O F_{r f}^{s i m}$. The calculated point represents the predicted travel-time value, TOF $\mathrm{Ff}_{\mathrm{rf}}^{\text {pred }}$, eq. (26). 


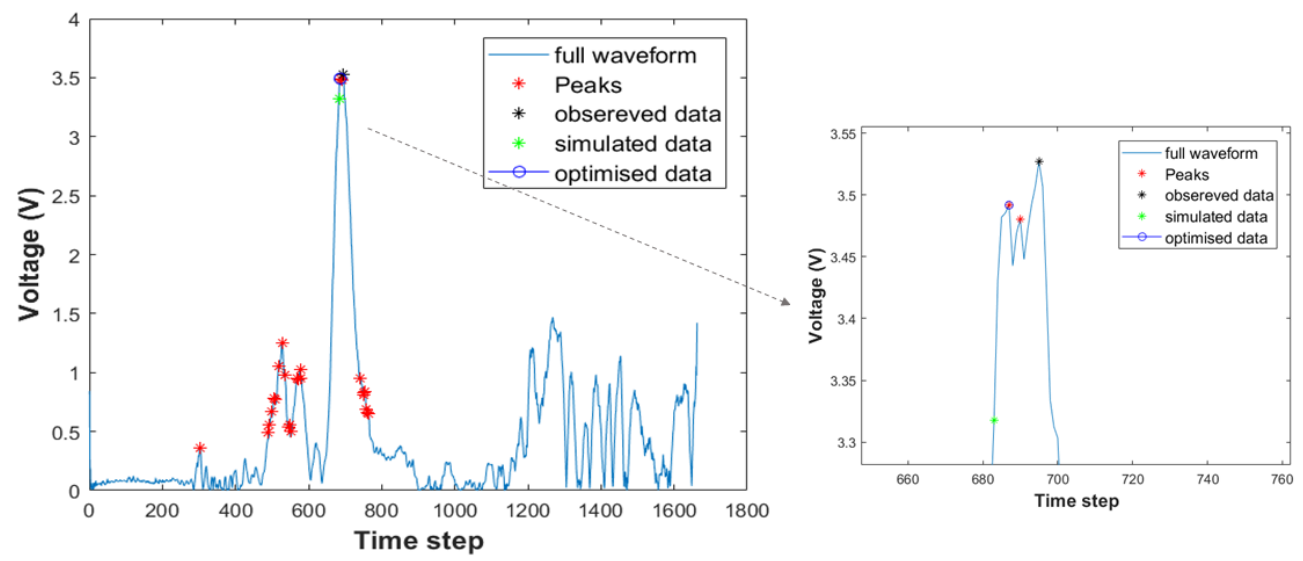

(a)

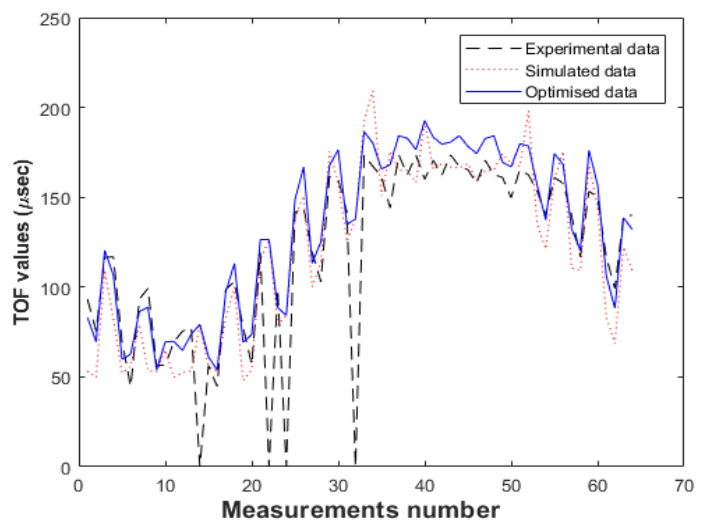

(b)

Figure 7. (a) Subtracted full-waveform signal with Peaks, observed, simulated, optimised data depicted. The corresponding pulse is represented zoomed. (b) The plot of experimental, simulated, and "optimal" data of all waveforms.

$$
T O \mathrm{~F}_{r f}^{\text {pred }}=P \mid \min _{n \varepsilon N}\left(\mathrm{P}-T O F_{r f}^{\text {sim }}\right)
$$

Where $P$ is a $m=[1, \ldots, M]$ by $n=[1, \ldots, N]$ matrix, containing the peaks of the full-waveforms; $M$ is the number of the measurements and $N$ the number of peaks for each measurement. Usually, the generated $T O \mathrm{~F}_{r f}^{\text {pred }}$ data were optimised compared to the observed ones, TOF $F_{r f}^{o b s}$. Nevertheless, the data were occasionally highly affected by the simulated data, leading to overfitting cases. Therefore, a polynomial least square fitting model was used between the observed reflection data and the "optimal" data, to smooth the data. A convergence criterion of the average percentage of similarity of the observed data, $T O F_{r f}^{o b s}$, and the predicted data, TOF $F_{r f}^{\text {pred }}$, was also used. Eq. (27) describes this criterion.

$$
C=\frac{\left|T O F_{r f}^{o b s}-T O F_{r f}^{\text {pred }}\right|}{T O F_{r f}^{o b s}} \text { with } C<0.1
$$

If the convergence criterion is true, then the optimisation is applied to the data. Such a technique is widespread in concepts of full-waveform inversion [44], [45], [46] The simulated data are used to optimise the already captured data, solving the cost function in eq. (27).

$$
\begin{gathered}
\varepsilon=\frac{1}{2} \sum_{m=1}^{M}\left[\operatorname{TOF}_{r f}^{o b s}(m)-\mathrm{TOF}_{r f}^{\text {pred }}(m)\right]^{2} \\
\xi_{o p t}=\arg \min \|\varepsilon(\xi)\|
\end{gathered}
$$

Where $\xi$ represents the acoustical property distribution to be recovered, and $\varepsilon(\xi)$ is the error functional. This final step produces the last reflection data $T O F_{r f}^{o p t}$, which is optimal. Figure 7(a) shows the difference data signal, computed by eq. (12), and a zoomed window of the reflection pulse. The black dot represents the travel-time of the reflected pulse coming from the straightforward method of section II.B (TOF $\left.F_{r f}^{o b s}\right)$. The green dot is the simulated travel-time, $T O F_{r f}^{s i m}$. The red dots represent all the captured peaks of the waveform above the threshold value. Finally, the blue circle represents the "optimal" reflected travel-time value that results from the "minimal distances method", TOF $\mathrm{F}_{\mathrm{rf}}^{\mathrm{opt}}$. The effect of the method is noticeable in Figure 7(b). The blue function represents the optimised data, there red function the simulated data and the black function the recorded data. 


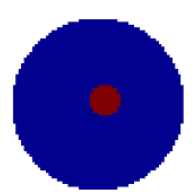

(a)

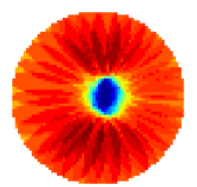

(b)

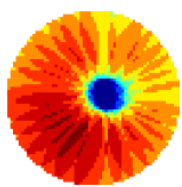

(c)

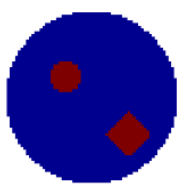

(d)

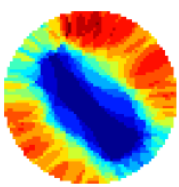

(e)

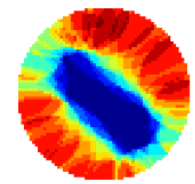

(f)

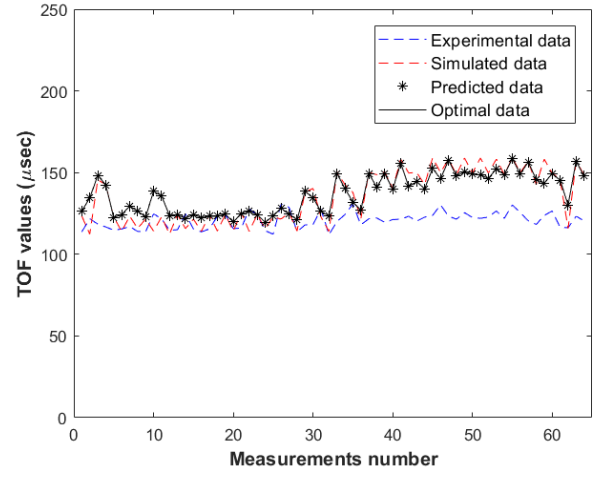

(g)

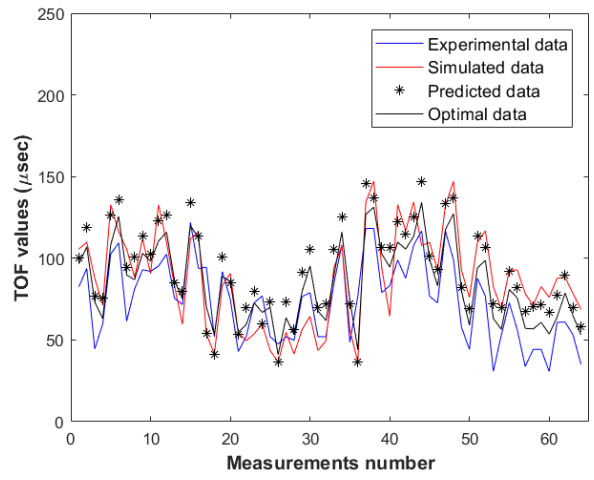

(h)

Figure 8. (a) True images of two tested configurations. (b), (d) Reflection images generated by the "traditional" method. (c), (e) Reflection images generated by the "proposed" method. (f) Plots of observed, simulated and optimal reflection data.

The black function missed calculating a correct TOF value in few cases, where zeros are observed. However, the zeros have been replaced with estimated values, in the optimised data, with the aid of simulated data, concluding in the well-behaviour of the optimization. These travel-time values are assumed to be refined when compared to the observed data. This method ensures a significantly richer dataset than the straightforward way of picking the reflected pulses. This method is named as "minimum distances method".

Figure 8 presents the reflection data optimisation process for two single and double inclusions cases. Specifically, Figure 8 (a), (d), show the true images, while Figures 8 (b)-(e) present reflection reconstructions using the straightforward "traditional" way0 for reflection TOF picking data from and "proposed" reflection TOF picking methods. Finally, figure 8 (f) presents the reflection TOF of observed, simulated and optimal data. The observed data come from the "traditional" method, the simulated data comes from the reflection forward solver, and the optimal one comes from the "proposed" method. Both two stages of "minimal distances" and "least-square fitting" were plotted for optimal data. In the first case, the two functions coming from optimal data are the same, which means that the convergence criterion is not valid. On the contrary, in the second case, these functions differ. In both cases, the effect of the simulated data for the computation of optimal data is pronounced. Optimal data seem to be a processed function dragged by the optimised ones.

The novel reflection data picking algorithm consists of all the previously described methods and aims to provide optimal reflection TOF data. The proposed algorithm fits the optimal data to the captured ones with respect to a priori information of the simulated data, as shown in Figure 7. It can be summarised into the following steps: (i) execution of transmission reconstruction; (ii) segmentation using Otsu's threshold; (iii) execution of reflection forward solver to produce simulated data; (iv) calculation of optimised reflection TOF by "minimum distances"; (v) checking the convergence criterion and if true, finding the misfit data $T O F_{r f}^{\mathrm{opt}}$ by solving the cost function for TOF $F_{r f}^{o b s}$ and TOF $F_{r f}^{\text {pred }}$. The whole method is displayed in Algorithm 1.

\begin{tabular}{|c|c|}
\hline \multicolumn{2}{|r|}{ ALGORITHM 1. Novel Reflection signal picking } \\
\hline 1 & $\begin{array}{l}\text { Compute } \mathbf{T O F} \text { rfl by using the "traditional" TOF picking } \\
\text { method. }\end{array}$ \\
\hline 2 & : $\quad$ Produce fused AA-TOF transmission image. \\
\hline 3 & $\begin{array}{l}\text { : Create the acoustic domain by segmentation of fused } \\
\text { transmission image. }\end{array}$ \\
\hline 4 & $\begin{array}{l}\text { Compute } \mathbf{T O} \mathbf{F}_{\mathbf{r f l}}^{\text {sim }} \text { by solving the reflection forward } \\
\text { problem. }\end{array}$ \\
\hline 5 & : Detect all waveform's peaks above a minimum threshold. \\
\hline 6 & $\begin{array}{l}\text { Calculate } \boldsymbol{T O F}_{\text {relf }}^{\text {pred }} \text { by locating the shortest distance } \\
\text { peaks from } \mathbf{T O F}_{\boldsymbol{r f l}}^{\text {sim }} \text { by using the minimum distance } \\
\text { method. }\end{array}$ \\
\hline 7 & : $\quad$ Calculate the average percentage of similarity, $C$. \\
\hline 8 & $: \quad$ If $(C<0.1)$ \\
\hline 9 & $\begin{array}{l}\text { Solve the cost function of } \boldsymbol{T} \boldsymbol{O F}_{\text {relf }}^{\text {pred }} \text { and } \\
\boldsymbol{T O F}_{\text {refl. }}^{\text {obs }}\end{array}$ \\
\hline 10 & : end \\
\hline
\end{tabular}

\section{TRIPLE MODALITY}

The developed triple modality approach consists of three sets of information, and the fusing method is described by a specific pipeline which is depicted in Figure 9. First, the TOF and AA transmission images are fused using a "wavelet transform method" [47]. This worked well due to the similarities of the TOF and AA images as they both come from transmission reconstruction. The two images were normalised, before being merged, to have the same scale. The result contains both the information coming from TOF and AA data subsequently 
proved as an optimised reconstructed image. Then, the transmission image is combined with the reflection image.

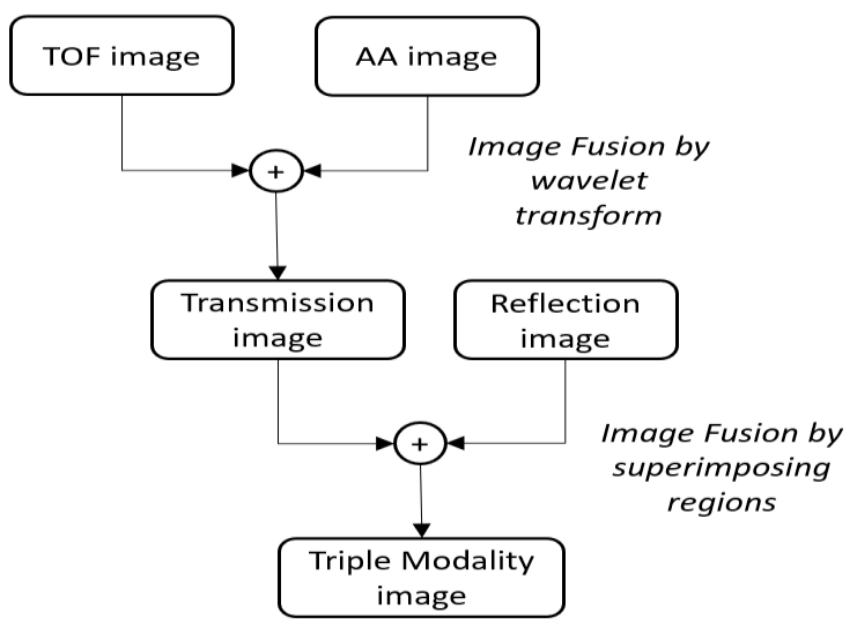

Figure 9. Image fusion algorithm for triple modality USCT.

Because of the different nature of the transmission and reflection images, a different fusion method was used. The transmission images usually contain high "regions" in the position where the objects are located, due to significantly high TOF-delays and amplitude attenuation due to object introduction. On the other hand, the reflection image has almost zero values to the locations of the objects, as all the reflections are encountered in their boundaries and, according to the ellipse algorithm, no ellipse interaction is happening within the object. Therefore, a method that accounts for these characteristics, by superimposing regions of the images, was applied to fuse the transmission and reflection images. This is described in eq. (30) method.

$$
\begin{gathered}
T M_{i, j}=\left\{\begin{array}{ll}
T_{i, j} & \text { where } R_{i, j}>0 \\
0 & \text { where } R_{i, j}=0
\end{array} \text { where },\right. \\
i=[1, \ldots, 32] j=[1, \ldots, 32]
\end{gathered}
$$

Where $T_{i, j}$ is the transmitted image, $R_{i, j}$ is the reflection image and $T M_{i, j}$ is the triple-modality image; $i, j$ represents the rows and columns of the image that is 32 by 32 .

\section{RESULTS AND ANALYSIS}

The system was experimentally validated by applying several single and multiple static inclusions tests with different shapes and sizes. All the inclusions are made from plastic (PVC) and are not compact; thus, the sound can only be diffracted and reflected. Circular inclusions of $1 \mathrm{~cm}, 2 \mathrm{~cm}$ and $3 \mathrm{~cm}$ of diameter, square inclusion of $4 \mathrm{~cm}$ side-length and an equilateral triangle inclusion of $3 \mathrm{~cm}$ were used to provide various testing cases. These tests aimed to simulate dispersed phases of a liquid mixture existing in industrial processes. The change in the structural phase aims to simulate the change happening during a crystallization or fermentation process. Figure 10 presents results using different reconstruction methods of 10 different experimental configurations. Among the reconstructed methods are TOF, AA, fused transmission, "traditional" reflection, "proposed" reflection and triplemodality reconstruction methods. It is evident that transmission mode has can be used in object localisation, notably upon multiple inclusions. On the other hand, reflection is significantly better in detecting the boundaries of the domain accurately. However, reflection has a clear disadvantage in reconstructing regions that lie between two objects. Therefore, in those cases, the transmission mode aid more the triplemodality results than the reflection.

To quantify the imaging quality of the proposed reconstruction approach, Correlation Coefficient (CC) and Root Mean Square Error (RMSE) were calculated, eq. (31) and eq. (32) respectively. The segmentation method described in section IV. Normalisation was applied to all the images to turn them into a uniform form, aiming at quantitative similarities.

$$
\begin{gathered}
C C=\frac{\sum_{n=1}^{N}\left(\sigma_{n}-\delta\right)\left(\sigma_{n}^{*}-\delta^{*}\right)}{\sqrt{\sum_{n=1}^{N}\left(\sigma_{n}-\delta\right)^{2} \sum_{n=1}^{N}\left(\sigma_{n}^{*}-\delta^{*}\right)^{2}}} \\
R M S E=\sqrt{\frac{\sum_{n=1}^{N}\left(\sigma-\sigma^{*}\right)^{2}}{N}}
\end{gathered}
$$

Where $\sigma$ is the calculated acoustic distribution by the reconstruction algorithms and $\sigma^{*}$ is the real one (true image), $\sigma_{n}$ and $\sigma_{n}^{*}$ are nth elements of $\sigma$ and $\sigma^{*}$ respectively, $\delta$ and $\delta^{*}$ are the mean values of $\sigma$ and $\sigma_{n}^{*}$, respectively. Figure 11 shows the $\mathrm{CC}$ and the RMSE of TOF, AA, fused transmission, "traditional" reflection, "proposed" reflection and triplemodality reconstructions. In almost all the cases, the proposed algorithm proved to be more efficient by acquiring the overall highest $\mathrm{CC}$ and lowest RMSE value. CC was higher and RMSE was lower in single inclusion cases comparing with the multiple inclusions cases due to the medium's complexity.

Concluding in the supremacy of the proposed reflection algorithm, a triple modality approach was applied using TOF, AA and reflection images. The MRSE of triple-mode images is generally smaller, while $\mathrm{CC}$ is generally more prominent than all the other methods. Although TOF and AA images converted to binary form using a high threshold to segment the inclusions. Then transmission and reflection images were fused in binary format. Regarding CC, in almost all cases, the final image is closer to the real geometry. The significant aid of the triple modality method can clearly be noticed as, in all cases, the TOF, AA and reflection reconstructions' accuracy differs, but the triple-modality reconstruction is always higher. The qualitative difference can be noticed between the single and multiple inclusions, coming from the more complex nature of the second case. The quantitative analysis indicates that the multi-modality method provides more accurate reconstruction on both the area and the location of the objects than a single modality of either transmission or reflection mode.

To further test the performance of the proposed system and the multi-modality approach, different setups of water/sucrose solutions were used. 


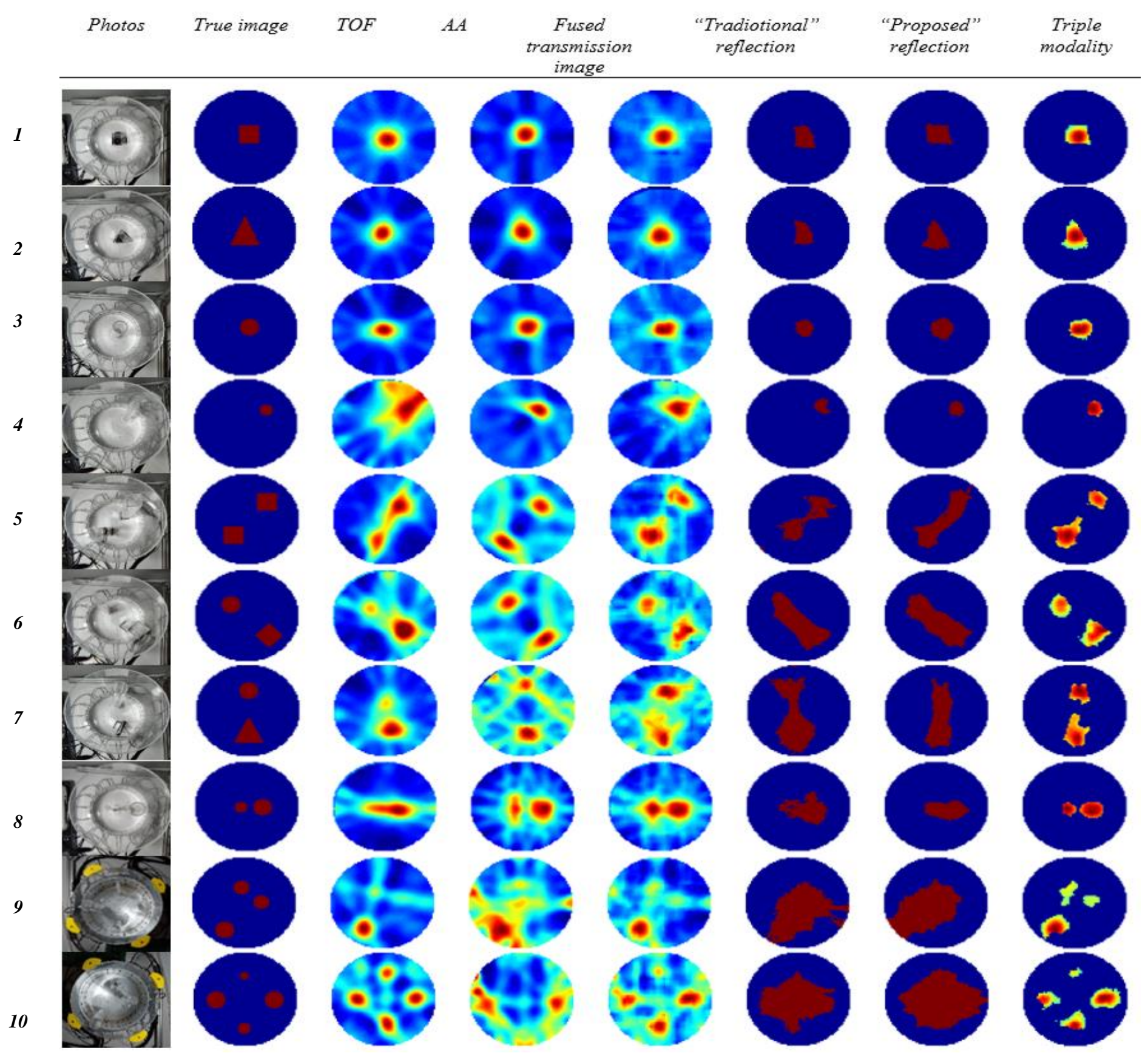

Figure 10. Image reconstructions of the Triple-Modality USCT.

These experimental scenarios simulate miscible liquids and multi-phase flow in industrial tanks and pipes scenarios. A plastic cup of $1 \mathrm{~mm}$ length, filled with the solutions, was used as a static inclusion. The cup's sound transmissivity has been tested and approved. The system recognised transmitted signals passed through the water/sucrose solutions and reflected signals coming from the cup's surface. Figure 12 displays experimental photos and reconstructions of three different water/sucrose cases. Figure 12(a) shows a $60.7 \%$ solution positioned in the centre, Figure 12(b) a combination of $50 \%$ and $42.8 \%$, and Figure 12(c) a combination of $20 \%$ and $42.8 \%$. The TOF mapping distinguished well between different concentrations in multiple inclusions experiments. Furthermore, the tests TOFdelays scale follows the overall concentration increase, as shown in Figure 12(b), (c). Additionally, six different single inclusions cases with water/sucrose concentrations of $20 \%$, $33 \%, 42.8 \%, 50 \%, 56.7 \%$ and $60.7 \%$ were reconstructed. Table 1 shows the TOF delays caused due to the existence of the solution. The presented TOF-delays, calculated as the object was segmented and its mean value was calculated. Difference imaging was used by subtracting the background TOF from the full TOF measurements. Since the sound velocity of the concentrations is higher than the medium (water in $20^{\circ} \mathrm{C}$ ), the produced difference data were negative. Small positive values were caused by noise and therefore were neglected. TOF delays showed good response, as they form an ascending function over the increasing concentration of the solutions. 


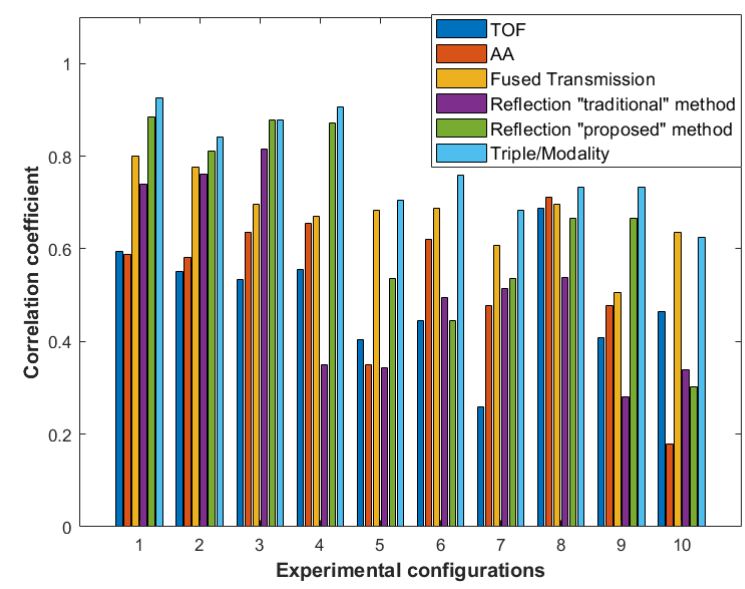

(a)

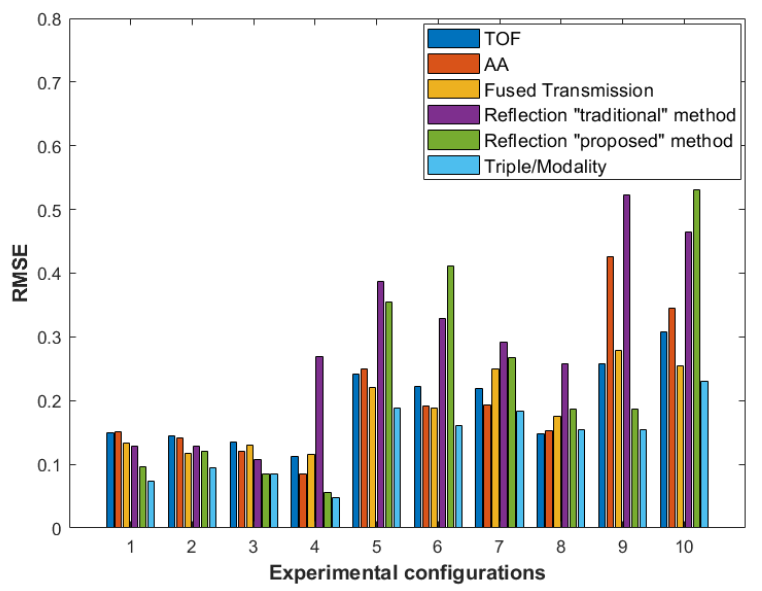

(b)

Figure 11. (a) CC and (b) RMSE of several different reconstruction methods.
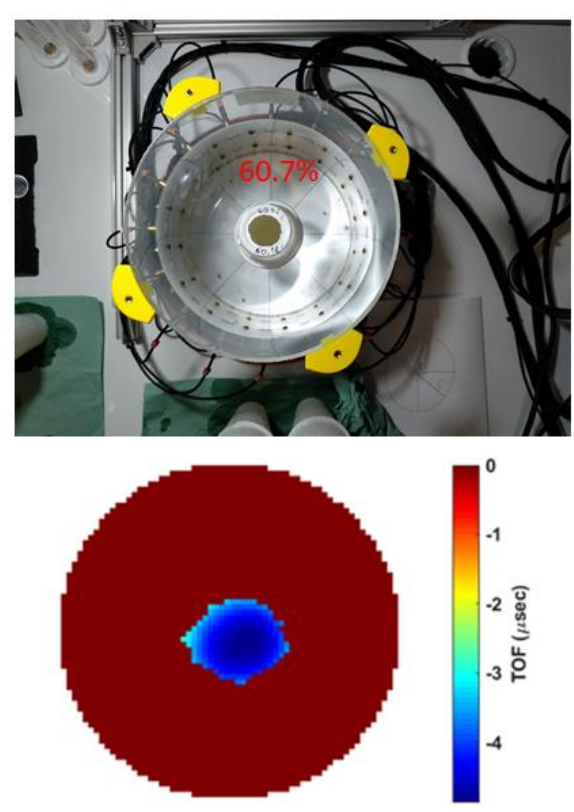

(a)
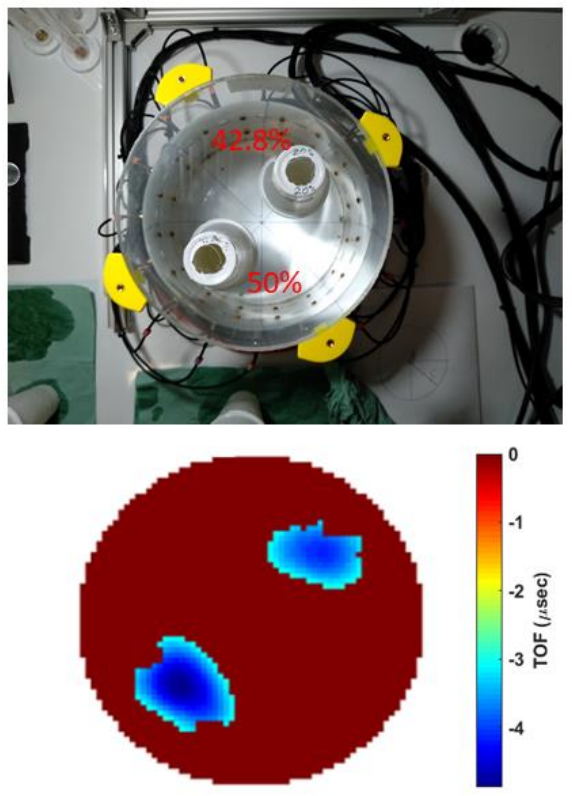

(b)
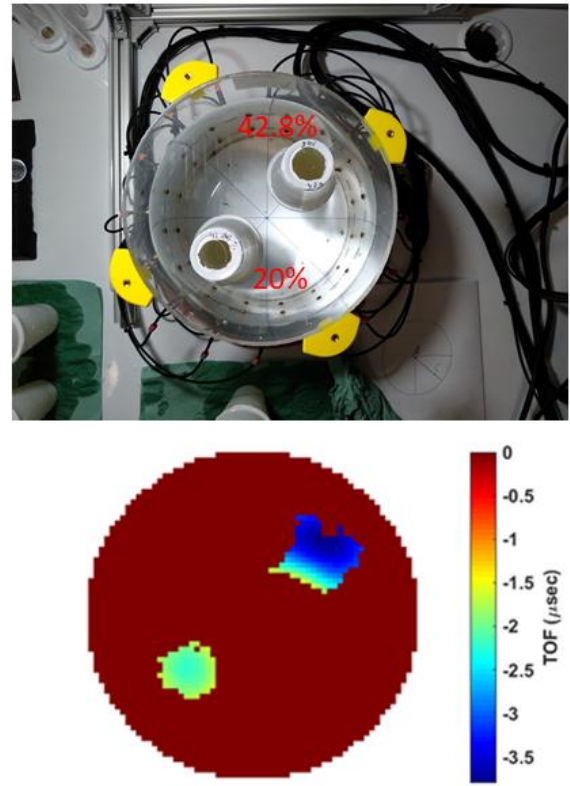

(c)

Figure 12. Experimental photos and reconstructions of water/sucrose solutions of (a) $60.7 \%$ in the centre (b) $50 \%$ down-left and $42.8 \%$ upright (c) 20\% down-left and $42.8 \%$ up-right.

The solutions experiment proved efficient in distinguishing between low changes of concentration, showing the high quantitative resolution that the system can provide.

TABLE I

TOF DELAYS FROM THE EXPERIMENTAL PROCESS WITH WATER/SUCROSE SOLUTIONS.

\begin{tabular}{cc}
\hline \hline $\begin{array}{c}\text { Mass concentration } \\
(\text { mass } / \text { volume })\end{array}$ & TOF delays \\
\hline $20 \% \mathrm{~m} / \mathrm{vol}$ & $1.94 \mu \mathrm{sec}$ \\
\hline $33 \% \mathrm{~m} / \mathrm{vol}$ & $2.85 \mu \mathrm{sec}$ \\
\hline $42.86 \% \mathrm{~m} / \mathrm{vol}$ & $3.69 \mu \mathrm{sec}$ \\
\hline $50 \% \mathrm{~m} / \mathrm{vol}$ & $3.96 \mu \mathrm{sec}$ \\
\hline $56.52 \% \mathrm{~m} / \mathrm{vol}$ & $4.14 \mu \mathrm{sec}$ \\
\hline $60.78 \% \mathrm{~m} / \mathrm{vol}$ & $4.42 \mu \mathrm{sec}$ \\
\hline
\end{tabular}

\section{CONCLUSIONS}

This work presents the advantages of triple-modality ultrasound tomographic imaging for real industrial processes. Accurate results of multiple solid objects and various concentrated solutions could significantly benefit complex industrial processes of multi-material interactions such as in two-phase media. Reflection and transmission reconstruction methods can work in a complementary way and provide optimal results. Moreover, acoustic attenuation measurements were proven effective, facilitating the transmission of TOF reconstructions, especially in more inhomogeneous media. So, there is great potential in the combination of two types of transmission mode tomography. This kind of rich fullwaveform tomography proved to work well in exploiting full- 
waveform information. Without introducing heavy computational algorithms, it can benefit from combining different reconstructions and at the same time perform at a high temporal frequency. Therefore, it comprises a potential solution to many industrial processes that need inspection over time and a good temporal resolution.

The developed methods provided good qualitative and quantitative performance regarding the quality image measurements and the correlation of TOF-delays with various solutions. Static experiments showed good system performance in distinguishing objects of different sizes and shapes in single and multiple objects. The solutions used in the experiments showed that the triple-modality imaging could also use the TOF scale to characterise small changes in the density of biphasic media, which is a significant addition to the system. The results of this research show that this rich full-waveform USCT can aid industrial processes and may be used for stirred tanks chemical processes. Given the existence of biphasic media, which include integration of liquid solutions and suspensions, the added value of the multi-modality full-waveform system will become apparent in our future studies.

\section{REFERENCES}

[1] H. I. Schlaberg, M. S. Beck, B. S. Hoyle, C. Lenn, and Ming Yang, "Real-time ultrasound process tomography for two-phase flow imaging using a reduced number of transducers," IEEE Trans. Ultrason. Ferroelectr. Freq. Control, vol. 46, no. 3, pp. 492-501, 2002.

[2] T. Rymarczyk and J. Sikora, "Applying industrial tomography to control and optimization flow systems," Open Phys., vol. 16, no. 1, pp. 332-345, 2018.

[3] L. Xu, Y. Han, L. A. Xu, and J. Yang, "Application of ultrasonic tomography to monitoring gas/liquid flow," Chem. Eng. Sci., vol. 52, no. 13, pp. 2171-2183, 1997.

[4] C. Tan, X. Li, H. Liu, and F. Dong, "An Ultrasonic Transmission/Reflection Tomography System for Industrial Multiphase Flow Imaging," IEEE Trans. Ind. Electron., pp. 11, Jan. 2019.

[5] R. Seidl, "Full Waveform Inversion for Ultrasonic Nondestructive Testing.", 2018.

[6] N. N. Kishore, I. Sridhar, and N. G. R. Iyengar, "Finite element modelling of the scattering of ultrasonic waves by isolated flaws," NDT E Int., vol. 33, no. 5, pp. 297-305, 2000.

[7] T. Rymarczyk, E. Kozłowski, G. Kłosowski, and K. Niderla, "Logistic Regression for Machine Learning in Process Tomography," Sensors, vol. 19, no. 15, p. 3400, 2019.

[8] H. I. Schlaberg, F. J. W. Podd, and B. S. Hoyle, "Ultrasound process tomography system for hydrocyclones," Ultrasonics, vol. 38, no. 1, pp. 813-816, 2000.

[9] J. Gu, H. Yang, F. Fan, and M. Su, "A transmission and reflection coupled ultrasonic process tomography based on cylindrical miniaturized transducers using PVDF films," J. Instrum., vol. 12, no. 12, Dec. 2017.

[10] Q. Su, C. Tan and F. Dong, "Measurement of phase fraction in oil-water two-phase flow using ultrasound attenuation method," 2015 Chinese Automation Congress (CAC), 2015, pp. 1890-1895,doi: 10.1109/CAC.2015.7382812.

[11] X. Duan, P. Koulountzios, and M. Soleimani, "Dual Modality EIT-UTT for Water Dominate Three-Phase Material Imaging," IEEE Access, vol. 8, pp. 14523-14530, 2020.

[12] Ming Yang, H. I. Schlaberg, B. S. Hoyle, M. S. Beck and C. Lenn, "Real-time ultrasound process tomography for two-phase flow imaging using a reduced number of transducers," in IEEE Transactions on Ultrasonics, Ferroelectrics, and Frequency Control, vol. 46, no. 3, pp. 492501, May 1999, doi: 10.1109/58.764834.

[13] G. T. Bolton and K. M. Primrose, "An overview of electrical tomographic measurements in pharmaceutical and related application areas," AAPS PharmSciTech, vol. 6, no. 2, 2005.

[14] H. Liu, C. Tan, S. Zhao, and F. Dong, "Nonlinear ultrasonic transmissive tomography for low-contrast biphasic medium imaging using continuous-wave excitation," IEEE Trans. Ind. Electron., vol. 67, no. 10, pp. 8878-8888, Oct. 2020.

[15] P. Koulountzios, T. Rymarczyk, and M. Soleimani, "A quantitative ultrasonic travel-time tomography to investigate liquid elaborations in industrial processes," Sensors (Switzerland), vol. 19, no. 23, 2019.

[16] Huang, L., Lin, Y., Williamson, M., 2014. Breast ultrasound waveform tomography: using both transmission and reflection data, and numerical virtual point sources, in: Medical Imaging 2014: Ultrasonic Imaging and Tomography. SPIE, p. 90400T. doi:10.1117/12.2043136

[17] G. Liang, S. Ren, and F. Dong, "Ultrasound guided electrical impedance tomography for 2D free-interface reconstruction,” Meas. Sci. Technol., vol. 28, no. 7, 2017.

[18] Li C, Huang L, Duric N, Zhang H, Rowe C. An improved automatic time-of-flight picker for medical ultrasound tomography. Ultrasonics. 2009 Jan;49(1):61-72. doi: 10.1016/j.ultras.2008.05.005. Epub 2008 Jun 7. PMID: 18620723 ; PMCID: PMC3915524.

[19] Y. Bao and J. Jia, "Improved time-of-flight estimation method for acoustic tomography system," IEEE Trans. Instrum. Meas., vol. 69, no. 4, pp. 974-984, 2020.

[20] L. Espinosa, J. Bacca, F. Prieto, P. Lasaygues, and L. Brancheriau, "Accuracy on the time-of-flight estimation for ultrasonic waves applied to non-destructive evaluation of standing trees: A comparative experimental study," Acta Acust. united with Acust., vol. 104, no. 3, pp. 429-439, 2018.

[21] Y. S. Huang, Y. P. Huang, K. N. Huang, and M. S. Young, "An accurate air temperature measurement system based on an envelope pulsed ultrasonic time-of-flight technique," Rev. Sci. Instrum., vol. 78, no. 11, 2007.

[22] H. I. Schlaberg, M. Yang, and B. S. Hoyle, "Ultrasound reflection tomography for industrial processes," Ultrasonics, vol. 36, no. 1-5, pp. 297-303, 1998.

[23] A. Macchi, H. Bi, J. R. Grace, C. A. McKnight, and L. Hackman, "Effect of gas density on the hydrodynamics of bubble columns and three-phase fluidized beds," Can. J. Chem. Eng., vol. 81, no. 3-4, pp. 846-852, 2003.

[24] E. Miller, B. T. Cox, J. L. Herraiz, J. M. Udías, M. Pérez-Liva, and B. E. Treeby, "Time domain reconstruction of 
sound speed and attenuation in ultrasound computed tomography using full wave inversiona)," J. Acoust. Soc. Am., vol. 141, no. 3, pp. 1595-1604, 2017.

[25] Alle, "Procedures for detecting outlying observations in samples," Technometrics, vol. 11, no. 1, pp. 1-21, 1969.

[26] C. Li, N. Duric, and L. Huang, "Comparison of ultrasound attenuation tomography methods for breast imaging," Med. Imaging 2008 Ultrason. Imaging Signal Process., vol. 6920, no. March 2008, p. 692015, 2008.

[27] P. Koulountzios, T. Rymarczyk, and M. Soleimani, "Ultrasonic time-of-flight computed tomography for investigation of batch crystallisation processes," Sensors (Switzerland), vol. 21, no. 2, pp. 1-19, Jan. 2021.

[28] M. L. Buursink, T. C. Johnson, P. S. Routh, and M. D. Knoll, "Crosshole radar velocity tomography with finitefrequency Fresnel volume sensitivities," Geophys. J. Int., vol. 172, no. 1, pp. 1-17, Jan. 2008.

[29] J. M. Jensen, B. H. Jacobsen, and J. ChristensenDalsgaard, "Sensitivity kernels for time-distance inversion," Sol. Phys., vol. 192, no. 1-2, pp. 231-239, 2000.

[30] T. M. Hansen, K. S. Cordua, M. C. Looms, and K. Mosegaard, "SIPPI: A Matlab toolbox for sampling the solution to inverse problems with complex prior information: Part 2Application to crosshole GPR tomography," Comput. Geosci., vol. 52, pp. 481-492, Mar. 2013.

[31] L. I. Rudin, S. Osher, and E. Fatemi, "Nonlinear total variation noise removal algorithm," Phys. D Nonlinear Phenom., vol. 60, no. 1-4, pp. 259-268, 1992.

[32] T. Goldstein and S. Osher, "The Split Bregman Method for L1-Regularized Problems," SIAM J. Imaging Sci., vol. 2, no. 2, pp. 323-343, 2009.

[33] F. Li, J. F. P. J. Abascal, M. Desco, and M. Soleimani, "Total Variation Regularization with Split Bregman-Based Method in Magnetic Induction Tomography Using Experimental Data," IEEE Sens. J., vol. 17, no. 4, pp. 976-985, 2017.

[34] J. F. P. J. Abascal et al., "Fluorescence diffuse optical tomography using the split Bregman method," Med. Phys., vol. 38, no. 11, pp. 6275-6284, 2011.

[35] L. Yang, C. Xu, and X. Guo, "Ring-shaped array ultrasound imaging using ellipse algorithm," Comput. Model. New Technol., vol. 18, pp. 315-320, 2014.

[36] J. Tu and B. Yang, "The Research of Ellipse Parameter Fitting Algorithm of Ultrasonic Imaging Logging in the Casing Hole,” Appl. Math., vol. 5, no. May, pp. 1317-1321, 2014.

[37] L. Yang, Q. Pan, C. Xu, X. Guo, and K. Peng, "Immersion ultrasonic reflection tomography by annular array system," in FENDT 2013 - Proceedings of 2013 Far East Forum on Nondestructive Evaluation/Testing: New Technology and Application, 2013, pp. 82-89.

[38] F. Wiegand and B. S. Hoyle, "Simulations for Parallel Processing of Ultrasound Reflection-Mode Tomography with Applications to Two-Phase Flow Measurement," IEEE Trans. Ultrason. Ferroelectr. Freq. Control, vol. 36, no. 6, pp. 652-660, 1989.

[39] L. Huang et al., "Breast ultrasound tomography with two parallel transducer arrays," in Medical Imaging 2016:
Physics of Medical Imaging, 2016, vol. 9783, p. 97830C.

[40] M. Williamson et al., "Breast ultrasound waveform tomography: using both transmission and reflection data, and numerical virtual point sources," Med. Imaging 2014 Ultrason. Imaging Tomogr., vol. 9040, p. 90400T, 2014.

[41] L. Brancheriau, P. Lasaygues, E. Debieu, and J. P. Lefebvre, "Ultrasonic tomography of green wood using a nonparametric imaging algorithm with reflected waves," Ann. For. Sci., vol. 65, no. 7, 2008.

[42] N. Otsu, "A Threshold Selection Method from GrayLevel Histograms,” IEEE Trans. Syst. Man. Cybern., vol. 9, no. 1, pp. 62-66, 1979.

[43] Haralick, Robert M., and Linda G. Shapiro, Computer and Robot Vision, Volume I, Addison-Wesley, 1992, pp. 2848.

[44] M. Perez-Liva, J. L. Herraiz, J. M. Udias, B. T. Cox, and B. E. Treeby, "Full-wave attenuation reconstruction in the time domain for ultrasound computed tomography," in Proceedings - International Symposium on Biomedical Imaging, 2016, vol. 2016-June, pp. 710-713.

[45] T. Alkhalifah and Y. Choi, "From tomography to fullwaveform inversion with a single objective function," Geophysics, vol. 79, no. 2, pp. R55-R61, 2014.

[46] R. Djebbi and T. Alkhalifah, "Traveltime sensitivity kernels for wave equation tomography using the unwrapped phase," Geophys. J. Int., vol. 197, no. 2, pp. 975-986, 2014.

[47] H. Li and B. s. Manjunath, "Multisensor-Image-FusionUsing-the-Wavelet-Transform_1995_Graphical-Models-andImage-Processing.pdf," Graphical models and image processing, vol. 57, no. 3. pp. 235-245, 1995.

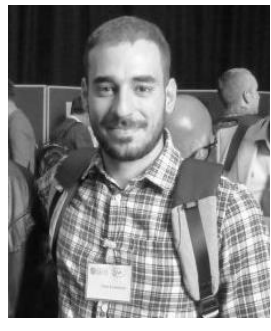

PANAGIOTIS KOULOUNTZIOS received the M.Eng. degree in electrical and computer engineering from the Technical University of Crete. He is currently pursuing the Ph.D. degree with the Engineering Tomography Laboratory (ETL). Since April 2018, he has been with ETL as Early Career Researcher (ESR) for Tomocon, an EU training network project. His Ph.D. is in ultrasound tomography for industrial process applications.

\section{TOMASZ RYMARCZYK}

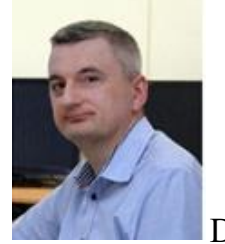

Director of Research and Development centre Netrix S.A. His research area focuses on application of noninvasive imaging techniques, electrical impedance tomography, image reconstruction and process tomography. Application of software engineering, AI and computerised measurement 
system is area of interest.

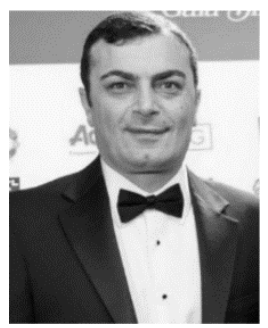

MANUCHEHR SOLEIMANI received the B.Sc. degree in electrical engineering and the M.Sc. degree in biomedical engineering, and the Ph.D. degree in inverse problems and electromagnetic tomography from The University of Manchester, Manchester, U.K., in 2005. From 2005 to 2007 , he was a Research Associate with the School of Materials, The University of Manchester. In 2007, he joined the Department of Electronic and Electrical Engineering, University of Bath, Bath, U.K., where he was a Research Associate and became a Lecturer, in 2008, a Senior Lecturer, in 2013, a Reader, in 2015, and a Full Professor, in 2016. In 2011, he founded the Engineering Tomography Laboratory (ETL), University of Bath, working on various areas of tomographic imaging, in particular multimodality tomographic imaging. He has authored or coauthored well over 300 publications in the field 